\title{
Studies on Photoacoustic Spectroscopy (PAS) of CNT NPs
}

Karunanithy $\mathbf{M}^{1,3}$, Nivetha $\mathbf{S}^{1,2}$, Ayeshamariam $\mathbf{A}^{1,2 *}$, Thirumamagal $\mathbf{R}^{4}$, Mohamed Saleem $\mathbf{A}^{1,5}$, Ahmed Ibraheem $\mathbf{B H}^{6}$ and Jayachandran $\mathbf{M}^{7}$

${ }^{1}$ Research and Development Center, Bharathidasan University, Thiruchirappalli, 620 024, India

${ }^{2}$ Department of Physics, Khadir Mohideen College, Adirampattinam, 614 701, India

${ }^{3}$ Department of Physics, SKPD Boys Higher Secondary School, Chennai, 600 001, India

${ }^{4}$ Research and Development Center, Bharathiar University, Coimbatore, 641046 , India

${ }^{5}$ Department of Physics, Jamal Mohamed College (Auto), Thiruchirappalli, 620 020, India

${ }^{6}$ Department of Mechanical Engineering, Syed Ammal Engineering College, Ramanathapuram, 623 501, India

${ }^{7}$ Department of Physics, Sethu Institute of Technology, Pullor, Kariapatti, 626 106, India

\begin{abstract}
The organic carbonaceous materials play an important role in governing the optical transport phenomena and bioavailability of nanomaterials. The nanomaterials established for the absorption of neutral organic compounds do not always apply to the facts and the importance of accounting for the particular sorption behavior of being increasingly recognized. This work carries out a study on the reaction of synthesis and optical and electrical characterizations of CNT carbon nanostrutures. The crystal size was calculated by using Scherer equation as $55 \mathrm{~nm}$ and its thermal diffusivity $4.515 \times 10^{-7} \mathrm{~m}^{2} / \mathrm{sec}$, the conductivity of the sample was measured as 75.69 . At the surface, the progressive incorporation of dopant triggers the diffusion of CNT to the surface, which results in fast formation carbon nanostructures of CNT, its crystallite size lies in the range of $36 \mathrm{~nm}$.
\end{abstract}

Keywords: Carbon nanostructure; Photo acoustic spectroscopy, CNT

\section{Introduction}

The nature of the support carbonaceous materials, the support structure (porosity, non-porosity), and the support surface properties (the functional groups present on the support surface) profoundly influence the morphology, dispersion, and electronic properties of the active phase (typically metallic). Surface properties of carbonaceous materials support, acidity/basicity to determine the catalytic activity, and many researchers have been published regarding the acidity of catalyst supports [1].

Lately discovered carbonaceous materials including CNTs showed promising candidate in a wide range of applications heterogeneous catalyst because they possess a large surface area, provide good mechanical strength and stable under harsh conditions. Nanomaterials are of large applications and they are effectively a bridge between bulk and atomic or molecular structures. It has constant physical properties regardless of its size, but at the nano-scale this is often not the case.

The recently discovered carbonaceous materials, including carbon nanotubes (CNTs) and graphene oxide (GO), show promise in a wide range of applications seeking to capitalize on their unique and fascinated properties [2]. CNTs, SWCNT and MWCNT are broadly employed as heterogeneous catalyst, because they possess a large surface area, provide good mechanical strength, and stable under harsh conditions. The carbonaceous material properties change as their size and as the percentage of atoms at the surface of a material becomes significant. For bulk materials larger than one micrometer, the percentage of atoms at the surface is very small relative to the total number of atoms of the materials [3]. The interesting and sometimes unexpected properties of nanoparticles are partly due to the aspects of the surface of the materials dominating the properties in lien of the bulk properties. In addition, the appropriate doping of $\mathrm{OH}$ groups is very important to enhance the materials properties and its applications. A little effort has been applied toward the use of other carbonaceous materials, such as GO [4] or CNTs [5] to study the photo acoustic properties of single sample.

Nanoparticles are constituted of several tens or hundreds of atoms or molecules and can have a variety of sizes and morphologies. Some kinds of Nanoparticles are already available commercially in the form of dry powders or liquid dispersions. The latter is obtained by combining Nanoparticles with an aqueous or organic liquid to form a suspension or paste. It may be necessary to use chemical additives (surfactants dispersants) to obtain a uniform and stable dispersion of particles with further processing steps, nanostructured powders and dispersions can be used to fabricate coatings components or devices that may or may not retain the nanostructure of the particulates raw materials [6].

Quantum confinement effect of nanostructured semiconductors having size close to Bhor radius [7] presently leads to the development of nano materials. These materials have large potentiality for photonic devices [8]. Transparent conducting oxides can be promising nano materials if their nanoclusters are suitably confined to generate excitons. Usually the excitons formed by the combination of electron and hole on exposure to electromagnetic radiation have photoluminescence property. The added advantage of these materials is visible transmissivity due to their wide band gap values [9]. Attempts have been made by various research groups [10] to study nanosized carbon structures such as CNT and its base materials. They have concentrated on the quantum confinement study of the nanostructured $\mathrm{CNT}$ with little emphasis on Indium tin oxide. Hence we have been interested in synthesizing nanoclustered CNT close to Bohr radius and characterizing photoluminescence behavior to understand its quantum confinement effect.

*Corresponding author: Ayeshamariam A, Research and Development Center Bharathiyar University, Coimbatore, 641046, India, Tel: +91 4565-241539; E-mail: aismma786@gmail.com

Received October 31, 2017; Accepted December 06, 2017; Published December 15,2017

Citation: Karunanithy M, Nivetha S, Ayeshamariam A, Thirumamagal R, Mohamed Saleem A, et al. (2017) Studies on Photoacoustic Spectroscopy (PAS) of CNT NPs. Fluid Mech Open Acc 4: 180. doi: 10.4172/2476-2296.1000180

Copyright: $\odot 2017$ Karunanithy M, et al. This is an open-access article distributed under the terms of the Creative Commons Attribution License, which permits unrestricted use, distribution, and reproduction in any medium, provided the original author and source are credited. 
The material thermal parameters and the processing of microelectronic devices [11] need investigations with thermal waves. Photo electronic technique is one of the tools used extensively for such characterization. In this case, the sample is usually irradiated by a modulated light beam, which is then absorbed by the material and converted heat. The heat diffuses to the sample surfaces and then into the surrounding gas of the photo acoustic cell. Finally, the thermal expansion of the gas generates the photo acoustic (PA) signal. It is known that PA signals are generated when modulated optical energy is absorbed by a crystalline sample as thermal waves generated in the sample. This would subsequently be observed as acoustic waves.

The physical interpretation of thermal waves produced in the photo-thermal experiments and the difference with the wave phenomenon have been discussed in detail elsewhere [12]. In the case of semiconductors, the photo acoustic signal provides us, besides the thermal properties, with additional information regarding the carriertransport properties. When the absorbed radiation (Photon energy) is greater than the band-gap energy, the excitation produces an excess carrier distribution in the semiconductor with energy above (below) the conduction (Valence) band. In a time scale of few picoseconds these photo injected carriers distribute the excess of energy between them via Coulomb interaction and finally this extra energy is given to the lattice by relaxing to the bottom (top) of the conduction (valence) band via the carrier-phonon interaction. As the excess carrier diffuses through the sample, the electron-hole pairs eventually recombine producing a second source of heat, which also diffuses into the semiconductor of CNT Powder. Diamond structures are produced from carbon rich materials such as coal, peat, lignite, petroleum pitch, wood, nutshells, etc., by the processes of carbonization and activation. It is one of the most popular adsorbents due to its cost efficiency, excellent adsorption ability, and acid/base- and thermo-stability.

The production, modification, application, adsorption, and generation, along with other aspects of gas sorption by carbon nanostrutures have been well studied. Biochar is a group of carbon materials produced from biomass by slow pyrolysis under inert atmosphere. Although the source materials and production methods of biochar are similar as carbon, the distinctions between them are obvious. Carbon nanotubes (CNTs) are engineered carbon nanomaterials in form of rolling up graphene sheets into cylinder. CNTs are usually produced by arc discharge. Graphene and its derivatives seem to be attractive alter-natives for toxic gas adsorption; however, the severe aggregation restricts their commercial applications as gas adsorbents.

A photo acoustic spectrum is a plot of the intensity of the acoustic signal detected by a microphone or a piezoelectric detector, against the excitation wavelength or another quantity related to the photon energy of the modulated excitation.

When the sample is crystalline, the thermal waves can be associated with lattice periodicity and when it goes amorphous, this thermal wave will be drastically affected and so there will be a drastic change in the PA signal [13].

\section{Experimental Section}

Carbon sphere support was synthesized using a hydrothermal method. Glucose was chosen as the carbon source. One teaspoon sugar and $5.0 \mathrm{~g}$ glucose were dissolved in $100 \mathrm{~mL}$ ultrapure water and then transfer into a $150 \mathrm{~mL}$ Teflon hydrothermal synthesized reactor, kept $150^{\circ} \mathrm{C}$ for the reaction of $6 \mathrm{~h}$. After that, the product was washed for three times with ultrapure water and kept warming at $200^{\circ} \mathrm{C}$ for $8 \mathrm{~h}$.
After that, $5 \mathrm{mmol}$ Single walled carbon nanotubes (SWCNTs) (outer diameter $<8 \mathrm{~nm}$, bundle length $10-30 \mathrm{~mm}$, purity $>95 \%$ ) were purchased from Sigma Aldrich Chemicals were added and keep stirring for $2 \mathrm{~h}$. Then $5 \mathrm{mmol} \mathrm{KMnO}_{4}$ and $20 \mathrm{~g}$ graphite powder were slowly added in the above solution and kept stirring for $60 \mathrm{~min}$. The above mixture then transferred into a Telfon-lined autoclave. The autoclave temperature was maintained at $100^{\circ} \mathrm{C}$ for $6 \mathrm{~h}$, and the obtained precipitates were washed with water and dried at $80^{\circ} \mathrm{C}$ for $12 \mathrm{~h}$. Filter and wash (using deionized water) the mixture using filter papers, collect the residue and dry overnight at room temperature in vacuum oven at $60^{\circ} \mathrm{C}$, we get very mg of CNT powder [14].

The photo acoustic spectroscopy measurement was performed with black carbon powder. This material can be considered a perfect absorber; in this way, the carbon measure serves to characterize the emission spectrum of the arc lamp. All the remaining measurements were normalized to the lamp spectrum. CNT sample was placed using a crucible with diameter $5 \mathrm{~cm}$; after that, sample were submitted to particular time at the stove ( 20 minutes) at $50^{\circ} \mathrm{C}$. Immediately after the pre-determined period, the sample was positioned at the photo acoustic cell and the photo acoustic spectroscopy measurement was performed. Light was mechanically modulated at $17 \mathrm{~Hz}$ or $70 \mathrm{~Hz}$ (depending on the measurement) and spectroscopic measurements were performed between $240 \mathrm{~nm}$ and $800 \mathrm{~nm}$; however, the results showed are limited to the region of the ultraviolet radiation $(\lambda<400 \mathrm{~nm})$, that is the region of interest for this study. Each spectrum was obtained and data was stored using a computer program developed at the photo thermal Laboratory at the IFGW/UNICAMP. After measurements were performed, data were analyzed later using 2005 the Origin 8.0 software, the experimental set up is shown in (Figure 1) [15].

Usually high electrical conductivity is necessary in order to minimize Joule heating, while a low thermal conductivity helps to retain heat at the junctions and maintain a large temperature gradient. In metals, the ratio of the thermal conductivity to electrical conductivity is a constant (Wiedemann-Franz-Lorenz law) and it is not possible to reduce one, while increasing the other. Metals possess Seebeck coefficients of $10 \mu \mathrm{V} /{ }^{\circ} \mathrm{C}$ or less, which are helpful for a large number of applications. In semiconductors the ratio of the thermal conductivity to electrical conductivity is greater than in metals because of their poorer electrical conductivity. This ratio can be decreased (i.e., the electrical conductivity can be increased) if the thermoelectric material is alloyed with an isomorphous element or compound. Thermal conductivity was calculated by using the general formula of the synthesized CNT [16]

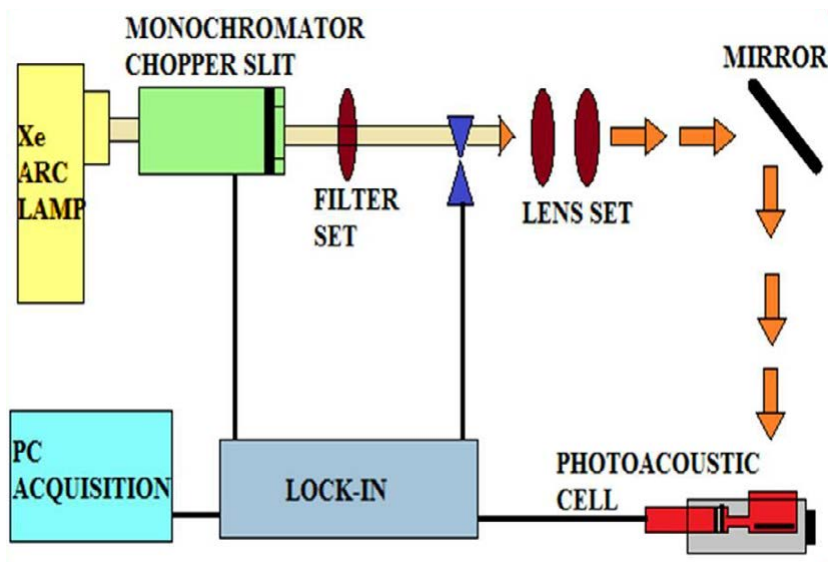

Figure 1: Photo acoustic spectroscopy experimental setup of the PA signal. 


\section{Results and Discussion}

Characteristic graphitic peak at $2 \theta=26.2^{\circ}$ referred to (002) exposes the perfect crystallinity of the CNT well matched with the results of JCPDS 26-10-77. This result is supported by the earlier work [17]. In $\mathrm{CNT}$, the carbons are coupled directly and form the hexagonal chain. Carbon by bond with positively charged carbons in the hexagonal frame as in the Figure 1. XRD signal pattern strongly represent the presence of weakly symbolize the CNT. The lattice parameter value of

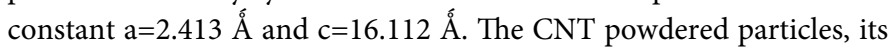
full width at half maximum (FWHM) $\beta$ is used in Scherer formula:

$$
D=\frac{0.9 \lambda}{\beta \cos \theta}
$$

The crystal size (D) and its related parameters are calculated for the compounds without and with annealing temperature of $200^{\circ} \mathrm{C}$. The variation in grain size is less appreciable and it decreases nominally about $36 \mathrm{~nm}$. From this observation, it can be noticed that, though thermal forces applied in the lattice sites, there is no considerable strain found and the structure does not changed.

The experimental section of Photo Acoustic Spectroscopy is shown in Figure 2. Thermal diffusivity is the rate of periodic heating or transient heat propagation through a medium. The thermal characteristics such as thermal diffusivity $(\alpha)$ of the sample affect the on a sample of thickness $l_{s}$ thermal conductivity $\mathrm{K}$ then the thermal diffusivity can be calculated from [17]:

$$
\alpha=f_{c} l_{s}^{2} \mathrm{~m}^{2} \sec ^{1}
$$

The value of $\alpha$ is a unique parameter for each material but is extremely dependent upon the compositional and microstructrual variables. The present study also verifies that the ion-implanted samples of $n$-CNT with different amount of doses have different values for the thermal diffusivity ( $\alpha$ ) due to the different levels of lattice damage.

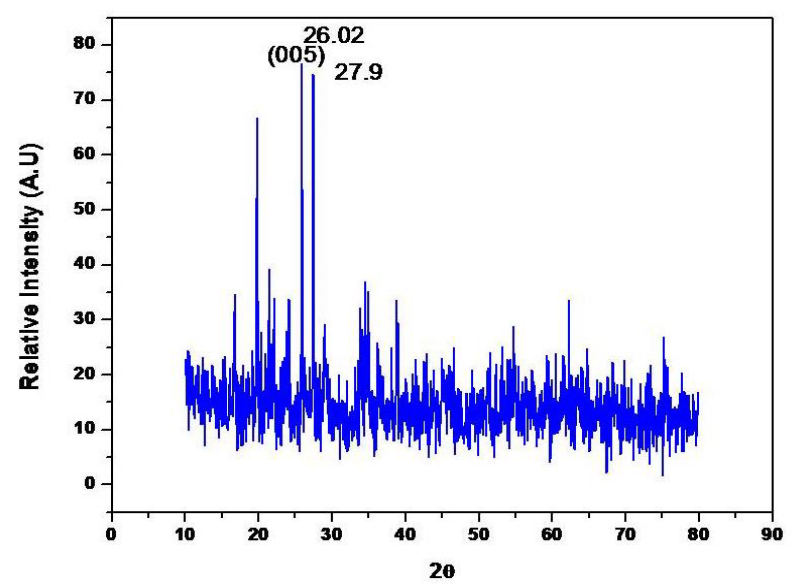

Figure 2: XRD analysis of carbon based material graphene oxide.
The thermal effusivity is:

$$
\mathbf{e}=\sqrt{f_{c} l_{s}^{l} \rho c_{p}{ }^{2}} \mathrm{Ws}^{1 / 2} / \mathrm{cm}^{2} \mathrm{~K}
$$

Where $\rho$ is the density, and $c_{p}$ is the specific heat capacity. Thermal conductivity is

$$
\mathrm{K}=\alpha \rho \mathrm{C}_{\mathrm{p}} \mathrm{W} / \mathrm{cm} / \mathrm{K}
$$

The density of the samples used and the specific heat capacity is and thickness of the samples for calculated values are listed in Table 1. Calculated values are listed in Table 2.

Thermal conductivity $(\mathrm{k})$, which is a kinetic property determined by the contributions from the vibrational, rotational, and electronic degrees of freedom, is an extremely important material property when high-power/high-temperature, electronic and optoelectronic devices are considered. For pure crystals, phonon-phonon scattering, which is ideally proportional to $\mathrm{T}^{-1}$ above the Debye temperature, is the limiting process for thermal conductivity. The heat transport is predominantly determined by phonon-phonon unklapp scattering, and phonon scattering by point and extended defects, such as vacancies (inclusive of lattice distortions caused by them), impurities, and point defects play a significant role in the thermal conductivity of CNT. The lattice contribution (phonon scattering) to the thermal conductivity $\mathrm{k}$ is obtained from the kinetic theory as

$$
k_{\text {lattice }}(T)=\frac{1}{3} V_{3} C_{\text {lattice }}(T) L(T),
$$

where $\mathrm{T}$ is the temperature, $\mathrm{V}_{\mathrm{s}}$ is the velocity of sound (almost independent of temperature), $\mathrm{C}_{\text {lattice }}(\mathrm{T})$ is the lattice specific heat, and $\mathrm{L}(\mathrm{T})$ is the phonon mean free path. In almost all materials $\mathrm{k}(\mathrm{T})$ first increases with temperature, reaches a maximum $\left(\mathrm{K}_{\max }\right)$ at some characteristic temperature $\mathrm{T}_{\mathrm{ch}}$, and then decreases. At low temperature $\mathrm{L}$ is relatively long and is dominated by extrinsic effects such as "defects" and/or finite crystal size and $C_{\text {lattice }}(T) \sim\left(T / \theta_{D}\right)^{3}$, where $\theta_{D}$ is the Debye temperature. As the temperature increases $\mathrm{C}_{\text {lattice }}(\mathrm{T})$ begins to saturate and intrinsic temperature-dependent umklapp processes become dominant, thus causing a decrease in L. The electronic contribution to the thermal conductivity which is negligible for carrier concentrations $\leq 10^{19} \mathrm{~cm}^{-3}$ is

$$
k_{\text {electr }}(T)=\frac{\pi^{2} n k_{B}^{2} T \tau_{\text {electr }}}{3 m_{c}^{*}}
$$

Where $\mathrm{n}$ is the carrier density, $\mathrm{K}_{\mathrm{B}}$ is the Boltzman constant, $\tau_{\text {electr }}$ is the scattering time of the electrons, and $m_{c}^{*}$ is the conduction band effective mass. The overall thermal conductivity generally decreases with increasing carrier concentration because the decrease in the lattice component of $\mathrm{k}$ due to increased phonon scattering from both the impurities and free electrons outweighs the increase in the electronic

\begin{tabular}{|c|c|c|c|c|c|c|}
\hline Sample & $f_{c(H Z)}$ & $I_{s(m) \times 10^{-4}}$ & $\rho \mathbf{k}_{\mathrm{gm} / \mathrm{m}^{3}}{ }^{3}$ & $C_{p j k g}{ }^{-1} k^{-1}$ & $K=\alpha_{s} \rho C_{p} w m^{-1} k^{-1}$ & $\mathbf{e}=\sqrt{f_{c} l_{s}^{2} \rho C_{p}^{2}} \mathbf{w s c m}{ }^{2} \mathbf{k} \times 10^{-4}$ \\
\hline CNT & 417.4 & 1.01 & 7.310 & 233 & 72.51 & 12999.0677 \\
\hline
\end{tabular}
contribution to $\mathrm{k}^{3}[18,19]$. The theory of Photo Acoustic Spectroscopy explains the variation of frequency, wavelength and normalized signal

\begin{tabular}{|c|c|c|c|c|c|c|}
\hline Sample & $\mathbf{f}_{\mathrm{c}(\mathrm{HZ})}$ & $I_{s}(m)$ & $\alpha=f_{c} I_{s}^{2} m^{2} / s e c$ & $\rho \mathrm{Kgm} / \mathrm{m}^{3}$ & $C_{\mathrm{p} \mathrm{JKg}}{ }^{-1} \mathrm{k}^{-1}$ & $K=\alpha_{s} \rho C_{p w} m^{-1} k^{-1}$ \\
\hline CNT & 373.2 & $1.10 \times 10^{-4}$ & $4.515 \times 10^{-7}$ & 7307.6 & 229.4 & 75.69 \\
\hline
\end{tabular}
graph shown in Figures 3 and 4 . It can be suggested that the main effect of the thermal conductivity, specific heat capacity and density of the sample was also calculated and reported in Table 2. Among the

Table 1: Calculated effusivity value of CNT.

Table 2: Thermal parameter value of CNT. 


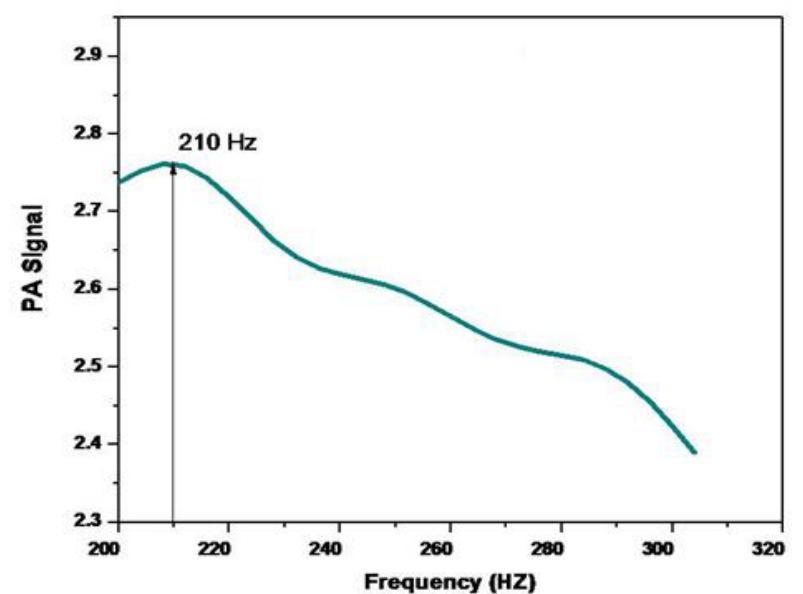

Figure 3: Variation of photo acoustic spectroscopy frequency with respect to PA signal.

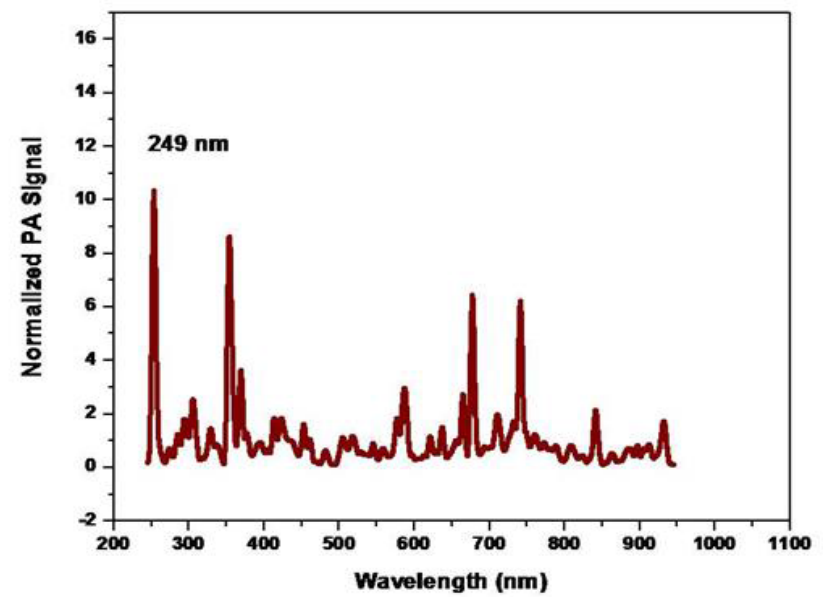

Figure 4: Variation of photo acoustic spectroscopy wavelength with respect to normalized PA signal.

carbonaceous used for the purpose of increasing the thermoelectric property of CNT, it has the optimum combination of high electrical conductivity $(\rho)$ and low thermal conductivity (k). Its ratio is nearly equivalent to the metals shown in Table 1.

\section{Conclusion}

The similarity in the behavior of the PA signals to that of the strain parameter $\varepsilon_{1}$ (from XRD) very clearly demonstrates the close relationship of the optical/acoustical characteristics even the microscope change in the diffusivity on CNT crystals can be seen and its thermal conductivity was increased which was measured by PA technique. We observe a slope change in the graph for the different composition of CNT gives variation in cutoff frequency. The thermal parameters evaluated from the PA signals also very closely agree with the available reported values, and many of our results from this investigation will add new data in literature, as many of them are not reported.

\section{References}

1. Schmidt E, Bucher C, Santarossa G, Mallat T, Gilmour R, et al. (2012) Fundamental insights into the enantioselectivity of hydrogenations on cinchonamodified platinum and palladium. Journal of Catalysis 289: 238-248.

2. Kim TY, Yokota M, Uchida T, Sugimura T (2009) Conditioning of Palladiumon-carbon $(\mathrm{Pd} / \mathrm{C})$ for Uniform Cinchonidine Modification to Perform Highly Enantioselective Hydrogenation. Catalysis Letters 131: 279-284.

3. Sugimura T, Ogawa H (2010) Acceleration of Enantioselective Hydrogenation of Olefins over $\mathrm{Pd} / \mathrm{C}$ by Cinchonidine as a Chiral Modifier. Comparison with Cinchonine, Pseudoenantiomer. Chemistry Letters 39: 232-233.

4. Szőri K, Puskás R, Szöllősi G, Bertóti I, Szépvölgyi J, Bartók M (2013) Palladium Nanoparticle-Graphene Catalysts for Asymmetric Hydrogenation. Catalysis Letters 143: 539-546.

5. Guan Z, Lu S, Li C (2014) Enantioselective hydrogenation of $\alpha, \beta$-unsaturated carboxylic acid over cinchonidine-modified Pd nanoparticles confined in carbon nanotubes. Journal of Catalysis 311: 1-5.

6. Rosencwaig A (1973) Photoacoustic spectroscopy of solids. Optics Communications 7: 305-308.

7. Murphy, Edward J, Setlur AA, Garcia F, Lyons RJ (2017) "Color stable redemitting phosphors." U.S. Patent 9: 698.

8. Eduardo JH, Ribeiro C, Giraldi TR, Longo E, Leite ER, et al. (2004) Photoluminescence in quantum-confined $\mathrm{SnO}_{2}$ nanocrystals: evidence of free exciton decay. Applied Physics Letters 10: 1745-1747.

9. Ayeshamariam A, Bououdina M, Sanjeeviraja C (2013) Optical, electrical and sensing properties of $\ln _{2} \mathrm{O}_{3}$ nanoparticles. Materials Science in Semiconductor Processing 16: 686-695

10. Feng G, Wang SF, Song CF, Lü MK, Qi YX, et al. (2003) Synthesis and luminescence properties of $\mathrm{SnO}_{2}$ nanoparticles." Chemical Physics Letters 372: $451-454$.

11. Gromaire-Mertz MC, Garnaud S, GonzalezA, Chebbo G (1999) Characterisation of urban runoff pollution in Paris. Water Science and Technology 39: 1-8.

12. Victoria G, Amo J, Soriano J, González-Lahoz J (1999) Sociodemographic and psychological variables influencing adherence to antiretroviral therapy. Aids 13 1763-1769.

13. Annane D, Sébille V, Charpentier C, Bollaert PE, François B, et al. (2002) Effect of treatment with low doses of hydrocortisone and fludrocortisone on mortality in patients with septic shock. Jama 288: 862-871.

14. Zhang Y, Sun X, Pan L, Li H, Sun Z, et al. (2009) Carbon nanotube-ZnO nanocomposite electrodes for supercapacitors. Solid State lonics 180: 15251528

15. Rompe PCB, Dos Anjos FS, Mansanares AM, da Silva EC, Acosta-Avalos D (2005) Characterization of human skin through photoacoustic spectroscopy." In Journal de Physique IV (Proceedings), EDP sciences125: 785-787.

16. Chen G, Dresselhaus MS, Dresselhaus G, Fleurial JP, Caillat T (2003) Recent developments in thermoelectric materials. International Materials Reviews 48: 45-66.

17. Gustafsson SE (1991) Transient plane source techniques for therma conductivity and thermal diffusivity measurements of solid materials. Review of scientific instruments 62: 797-804.

18. Fujii M, Zhang X, Xie H, Ago H, Takahashi K, et al. (2005) Measuring the thermal conductivity of a single carbon nanotube. Physical review letters 95: 065502.

19. Lu L, Yi W, Zhang DL (2001) $3 \omega$ method for specific heat and therma conductivity measurements. Review of scientific instruments 72 : 2996-3003. 